\title{
ACCURACY OF THE FORMULAS FOR THE RATIO, REGULATION, AND PHASE ANGLE OF TRANSFORMERS
}

\author{
By P. G. Agnew and F. B. Silsbee
}

There has been a great deal of discussion of the formulas for the predetermination of the performance of transformers from the constants obtained from the "short circuit" and "open circuit" tests, particularly in regard to the formula for computing regulation. We have very little data available, however, which will enable one to compare the formulas with accurately observed values. Bedell ${ }^{1}$ and Drysdale ${ }^{2}$ have published results for some of the formulas, which show a very fair agreement but so far as the authors are aware no comparisons have hitherto been published based on the precise potentiometer methods now available for the direct determination of ratio, regulation, and phase angle.

\section{DERIVATION OF FORMULAS}

The derivation of these formulas is given below in rather extended form in order to show the magnitude of the approximations which are introduced. These approximations will be considered in greater detail than might seem necessary, but on account of the confusion which has arisen from the use of incorrect formulas for regulation, some of the larger central stations have requested such a detailed discussion. A derivation of the formula for regulation has been published by one of the authors in conjunction with Dr. M. G. Lloyd. ${ }^{3}$ As this formula may be developed with only a slight amount of extra work while deriving

\footnotetext{
${ }^{1}$ Bedell, Chandler, and Sherwood: Elect. World, 30, p. 190; Aug. 14, 1897.

2 Drysdale: Electrician, 65, p. 643: July, roro.

3 Lloyd and Agnew: This Bulletin, 6, No. 2, p. 273; 1909. Reprint No. 129.
} 
those for ratio and phase angle, its derivation is given here in a slightly more rigorous form.

Let $N=$ ratio of turns, primary to secondary.

$E_{1}, E_{2}, E^{\prime}=$ primary and reversed secondary terminal voltages, and primary induced voltage, respectively. $R_{1}, X_{1}, Z_{1}=$ primary resistance, reactance and impedance. $R_{2}, X_{2}, Z_{2}=$ secondary resistance, reactance and impedance.

$R, X, Z=$ total resistance, reactance and impedance, referred to the primary, so that

$$
R=R_{1}+N^{2} R_{2}, \quad X=X_{1}+N^{2} X_{2}, \quad Z=Z_{1}+N^{2} Z_{2}
$$

$I=\frac{I_{2}}{N}=$ load current referred to primary side.

$\theta=$ angle by which $I$ lags behind $E_{2}$.

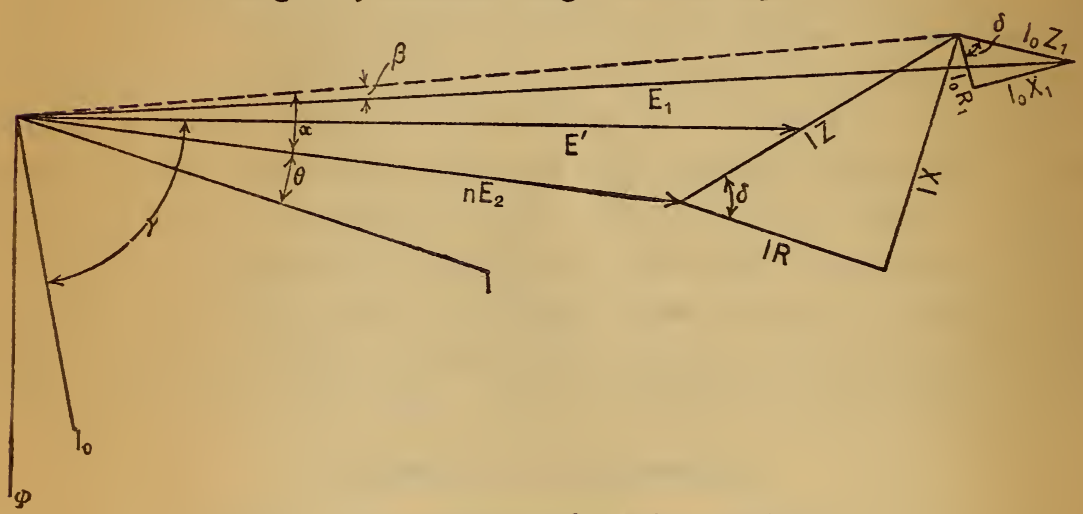

Fig. 1.--Vector diagram of transformer voltages

$I_{0}, I_{0}-\Delta I_{0}=$ exciting current at no load and at full load, respectively.

$\phi=$ flux.

$\gamma, \gamma+\Delta \gamma=$ angle by which the exciting current lags behind the induced voltage at no load and at full load, respectively.

$\alpha=$ part of phase displacement caused by load current.

$\beta=$ part of phase displacement caused by exciting current.

$$
\delta=\tan ^{-1} \frac{X}{R}
$$

Then in Fig. I the vector difference between $E_{1}$ and $N E_{2}$ is made up of four parts, $I R$ and $I X$ in phase and in quadrature, respec- 
tively, with $I$, and $I_{0} R_{1}$ and $I_{0} X_{1}$ in phase and in quadrature, respectively, with $I_{0}$.

It is necessary to assume $X_{1}=N^{2} X_{2}$, since the reactances of the individual windings can not be directly determined independently. We shall assume $\frac{X_{1}}{R_{1}}=\frac{X_{2}}{R_{2}}=\frac{X}{R}$, but only in those terms which contain the exciting current, and as will appear later these whole terms are so small as to be negligible in most practical work.

In fact, it may be shown without trouble that if the whole analysis is carried through, assuming that $X_{1}$ and $N^{2} X_{2}$ have any given ratio, and carrying all approximations to the same order as is done in what follows, that the final formulas will not be affected in any way. We may then take $\mathrm{E}^{\prime}$ as terminating at the midpoint of $I Z$, and practically bisecting $\alpha$.

Expressing all vectors in the usual complex notation, and using dotted capitals to indicate complex quantities, we see from Fig. I

$$
\begin{aligned}
\dot{E}_{1} & =E_{1}[\cos (\alpha-\beta)+j \sin (\alpha-\beta)] \\
\dot{E}_{2} & =E_{2} \\
\dot{I} & =I[\cos \theta-j \sin \theta]
\end{aligned}
$$

and also

$$
\dot{Z}=Z[\cos \delta+j \sin \delta]=R+j X
$$

At no load we have

and with load

$$
\dot{I}_{o}=I_{o}[\cos \gamma-j \sin \gamma]
$$

$$
\frac{\cdot}{I_{0}-\Delta I_{o}}=\left(I_{o}-\Delta I_{o}\right)\left[\cos \left(\gamma+\Delta \gamma-\frac{\alpha}{2}\right)-j \sin \left(\gamma+\Delta \gamma-\frac{\alpha}{2}\right)\right]
$$

Then we have as our fundamental relation

$$
\begin{gathered}
N \dot{E}_{2}+\ddot{I} \dot{Z}+\overline{\left(I_{0}-\Delta I_{0}\right)} \dot{Z}_{1}-\dot{E}_{1}=0 \\
\therefore N E_{2}+I Z\{\cos \theta-j \sin \theta\}\{\cos \delta+j \sin \delta\} \\
+\left(I_{o}-\Delta I_{0}\right) Z_{1}\left\{\cos \left(\gamma+\Delta \gamma-\frac{\alpha}{2}\right)-j \sin \left(\gamma+\Delta \gamma-\frac{\alpha}{2}\right)\right\}\{\cos \delta+j \sin \delta\} \\
-E_{1}\{\cos (\alpha-\beta)+j \sin (\alpha-\beta)\}=0
\end{gathered}
$$


or

$$
\begin{gathered}
N E_{2}+I Z\{\cos (\theta-\delta)-j \sin (\theta-\delta)\} \\
+\left(I_{o}-\Delta I_{o}\right) Z_{1}\left\{\cos \left(\gamma+\Delta \gamma-\frac{\alpha}{2}-\delta\right)-j \sin \left(\gamma+\Delta \gamma-\frac{\alpha}{2}-\delta\right)\right\} \\
-E_{1}\{\cos (\alpha-\beta)+j \sin (\alpha-\beta)\}=0
\end{gathered}
$$

Separating real and imaginary parts

$$
\begin{aligned}
N E_{2}+I Z \cos (\theta-\delta) & +\left(I_{o}-\Delta I_{0}\right) Z_{1} \cos \left(\gamma+\Delta \gamma-\frac{\alpha}{2}-\delta\right) \\
& -E_{1} \cos (\alpha-\beta)=0 \\
I Z \sin (\theta-\delta)+ & \left(I_{0}-\Delta I_{0}\right) Z_{1} \sin \left(\gamma+\Delta \gamma-\frac{\alpha}{2}-\delta\right) \\
+ & E_{1} \sin (\alpha-\beta)=0
\end{aligned}
$$

In order to put these equations into usable form, further approximations must be made. In estimating the magnitude of these we may consider two cases:

Case 1. -When the impedance drop is 4 per cent of rated voltage and the magnetizing current is ro per cent of rated load current. This is a more unfavorable condition than is ordinarily found in practice. Here $\frac{I Z}{N E_{2}}=0.04$ and $\frac{I_{0} Z_{1}}{N E_{2}}=0.002$

Case 2.-When the impedance drop is 2.5 per cent of rated voltage and the exciting current is 6 per cent of rated current. This would cover the great majority of practical cases. Here

$$
\frac{I Z}{N E_{2}}=0.025 \text { and } \frac{I_{0} Z_{1}}{N E_{2}}=0.00075
$$

In evaluating the approximations, sines and cosines of the various angles will be taken as unity, in order that the values found may be limiting ones, even under the most unfavorable conditions.

Consider first the magnitude of the term

$$
\left(I_{0}-\Delta I_{0}\right) Z_{1} \cos \left(\gamma+\Delta \gamma-\frac{\alpha}{2}-\delta\right)
$$

in comparison with $\mathrm{E}_{1}$. Neglecting for the moment $\Delta I_{0}$ and $\Delta \gamma$, the term can not exceed 0.2 per cent of $E_{1}$ for Case I or 0.08 per cent for Case 2. The actual values of $\Delta I_{0}$ and $\Delta y$ require a 
determination of the rate of variation of $I_{0}$ and $\gamma$ with $E_{1}$, measurements which are not ordinarily made. In Case I $\Delta I_{o}$, the change in exciting current from full load to no load, would probably not be greater than Io per cent of $I_{o}$, so that the part of the term depending on $\Delta I_{0}$ would not exceed 0.02 per cent. For Case 2 the corresponding values would be 6 per cent and 0.005 per cent; that is, it is less than the errors of commercial measurement. A numerical analysis leads to the same conclusion in regard to the effect of $\Delta \gamma$ in both equations 1 and 2.

Hence both $\Delta I_{0}$ and $\Delta \gamma$ may be neglected and the formulas take the form

$$
\begin{gathered}
N E_{2}+I Z \cos (\theta-\delta)+I_{0} Z_{1} \cos \left(\gamma-\frac{\alpha}{2}-\delta\right)-E_{1} \cos (\alpha-\beta)=0 \\
I Z \sin (\theta-\delta)+I_{0} Z_{1} \sin \left(\gamma-\frac{\alpha}{2}-\delta\right)+E_{1} \sin (\alpha-\beta)=0
\end{gathered}
$$

For no load

$$
\begin{gathered}
N E_{2,0}+I_{0} Z_{1} \cos (\gamma-\delta)-E_{1} \cos \beta=0 \\
I_{0} Z_{1} \sin (\gamma-\delta)-E_{1} \sin \beta=0
\end{gathered}
$$

From (3)

Ratio $=\frac{E_{1}}{E_{2}}=\frac{N}{\cos (\alpha-\beta)}\left\{\mathrm{I}+\frac{I Z \cos (\theta-\delta)}{N E_{2}}+I_{0} Z_{1} \cos \frac{\left(\gamma-\frac{\alpha}{2}-\delta\right)}{N E_{2}}\right\}$

From (4) and (6) we have approximately

$$
\begin{aligned}
\alpha-\beta & =\sin (\alpha-\beta)=\frac{\mathrm{I}}{E_{1}}\left[-I Z \sin (\theta-\delta)-I_{0} Z_{1} \sin \left(\gamma-\frac{\alpha}{2}-\delta\right)\right] \\
\beta & =\sin \beta \quad=\frac{\mathrm{I}}{E_{1}} I_{o} Z_{1} \sin (\gamma-\delta)
\end{aligned}
$$

Adding (8) and (9) and neglecting a term of the form $I_{0} Z_{1} \cos (\gamma-\delta) \sin \frac{\alpha}{2}$ which in Case I could not exceed 0.004 per cent or 0.08 minute, we have

$$
\alpha=-\frac{\mathrm{I}}{E_{1}} I Z \sin (\theta-\delta)
$$


Expanding the term $\cos (\alpha-\beta)$ in (7) and neglecting squares of $\beta$ and cubes of $\alpha$ (since these are of the order 0.0004 per cent and 0.0003 per cent) and again neglecting the term $\frac{\alpha}{2}$ in the last term of $(7)$ since this is equivalent to the approximation just justified in (Io) we have

$$
\frac{E_{1}}{E_{2}}=N\left\{\mathrm{I}+\frac{(\alpha-\beta)^{2}}{2}+\frac{I Z \cos (\theta-\delta)}{N E_{2}}+\frac{I_{0} Z_{1} \cos (\gamma-\delta)}{N E_{2}}\right\}
$$

or, by (8), retaining only squares of $\alpha$

$$
\frac{E_{1}}{E_{2}}=N\left\{1+\frac{I Z \cos (\theta-\delta)}{N E_{2}}+\frac{I^{2} Z^{2} \sin ^{2}(\theta-\delta)}{2 E_{1}{ }^{2}}+\frac{I_{0} Z_{1} \cos (\gamma-\delta)}{N E_{2}}\right\}
$$

For regulation we have from (3) and (5)

$$
\begin{gathered}
N\left(E_{2,0}-E_{2}\right)=I Z \cos (\theta-\delta)+I_{o} Z_{1}\left\{\cos \left(\gamma-\frac{\alpha}{2}-\delta\right)-\cos (\gamma-\delta)\right\} \\
-E_{1}\{\cos (\alpha-\beta)-\cos \beta\} \\
=I Z \cos (\theta-\delta)+2 I_{o} Z_{1} \sin \left(\gamma-\frac{\alpha}{4}-\delta\right) \sin \frac{\alpha}{4} \\
\quad+2 E_{1} \sin \frac{(\alpha-2 \beta)}{2} \sin \frac{\alpha}{2}
\end{gathered}
$$

Regulation $=\frac{E_{2,0}-\mathrm{E}_{2}}{\mathrm{E}_{2}}$

$$
\begin{aligned}
= & \frac{\mathrm{I}}{N E_{2}}\left[I Z \cos (\theta-\delta)+2 I_{0} Z_{1} \sin \left(\gamma-\frac{\alpha}{4}-\delta\right)\left(\frac{\alpha}{4}-\frac{\alpha^{3}}{384}-\right)\right. \\
& \left.\left.+2 E_{1} \mid \frac{\alpha-2 \beta}{2}-\frac{(\alpha-2 \beta)^{3}}{48}-\right\}\left\{\frac{\alpha}{2}-\frac{\alpha^{3}}{48}-\right\}\right]
\end{aligned}
$$

It will finally appear that the first term in the brackets gives the regulation to $0 . I$ per cent and all other terms but one are negligible. Hence, as already shown for ratio, we may neglect squares of $\beta$, cubes of $\alpha$, the small angle $\frac{\alpha}{4}$ in the expression $I_{0} Z_{1} \sin \left(\gamma-\frac{\alpha}{4}-\delta\right)$, and put $\mathrm{NE}_{2}$ equal to $\mathrm{E}_{1}$ in the latter terms, without the error exceeding o.or per cent. Substituting values 
of $\alpha$ and $\beta$ from (8), (9), and (10) in (12) and making these approximations we have,

$$
\begin{gathered}
\text { Regulation }=\frac{I Z \cos (\theta-\delta)}{N E_{2}}+\frac{I^{2} Z^{2} \sin ^{2}(\theta-\delta)}{2 N^{2} E_{2}^{2}} \\
+\frac{I Z I_{0} Z_{1} \sin (\gamma-\delta) \sin (\theta-\delta)}{2 N^{2} E_{2}{ }^{2}}
\end{gathered}
$$

In Case $\mathrm{I}$ the last term is only 0.004 per cent, and so this also may be omitted.

\section{SUMMARY OF FORMULAS}

For convenience of reference the formulas for ratio, regulation, and phase angle are here grouped together for various conditions of loading. They are obtained from equations (II), (I 3 ), and (8) by means of the relations

$$
X=Z \sin \delta \text { and } R=Z \cos \delta
$$

In the derivation, to avoid confusion $\alpha$ was taken positive when the phase relations were as shown in figure $r$. In the final formulas given below, however, the sign has been changed so that the phase angle is positive when the reversed secondary voltage leads the primary. When \pm signs occur the upper sign should be used for lagging and the lower for leading secondary current.

$$
\begin{aligned}
\text { Ratio }=N[\mathrm{I} & +\frac{I R \cos \theta \pm I X \sin \theta}{N E_{2}}+\frac{(I R \sin \theta \mp I X \cos \theta)^{2}}{2 N^{2} E_{2}{ }^{2}} \\
& \left.+\frac{I_{0} R_{1} \cos \gamma+I_{0} X_{1} \sin \gamma}{N E_{2}}\right]
\end{aligned}
$$$$
\underset{(\mathrm{P} . \mathrm{F} .=\mathrm{I})}{\text { Ratio }}=\mathrm{N}\left[\mathrm{I}+\frac{I R}{N E_{2}}+\frac{I^{2} X^{2}}{2 N^{2} E_{2}{ }^{2}}+\frac{I_{0} R_{1} \cos \gamma+I_{0} X_{1} \sin \gamma}{N E_{2}}\right]
$$$$
\underset{\text { (no load) }}{\text { Ratio }}=N\left[\mathrm{I}+\frac{I_{0} R_{1} \cos \gamma+I_{0} X_{1} \sin \gamma}{N E_{2}}\right]
$$

Per cent regulation $=\mathrm{IOO}\left[\frac{I R \cos \theta \pm I X \sin \theta}{N E_{2}}+\frac{(I R \sin \theta \mp I X \cos \theta)^{2}}{2 N^{2} E_{2}{ }^{2}}\right]$

$\begin{aligned} & \text { Per cent regulation } \\ & (\mathrm{P} . \mathrm{F} .=\mathrm{I})\end{aligned}=\mathrm{I} \circ\left[\frac{I R}{N E_{2}}+\frac{I^{2} X^{2}}{2 N^{2} E_{2}^{2}}\right]$

$$
27647^{\circ}-14 \longrightarrow
$$


Phase angle $=\frac{\mathrm{I}}{E_{1}}\left[I R \sin \theta-I X \cos \theta+I_{0} R_{1} \sin \gamma-I_{0} X_{1} \cos \gamma\right]$

$\underset{(\mathrm{P} . \mathrm{F} .=\mathrm{I})}{\text { Phase angle }}=\frac{\mathrm{I}}{E_{1}}\left[-I X+I_{0} R_{1} \sin \gamma-I_{0} X_{1} \cos \gamma\right]$

$\underset{\text { (no load) }}{\text { Phase angle }}=\frac{\mathrm{I}}{E_{1}}\left[I_{o} R_{1} \sin \gamma-I_{o} X_{1} \cos \gamma\right]$

(These last three equations give the phase angle in radians. To get minutes, multiply by 3438 .)

For most practical work the square terms in the formulas for ratio and regulation may well be omitted.

\section{DISCUSSION OF FORMULAS}

The combined approximations made in the deduction of these formulas are less than o.or per cent in ratio and regulation, or 0.4 minute in phase angle in Case 2. For Case $I$ the approximations may under some conditions add up to 0.03 or 0.04 per cent in ratio and regulation or $\mathrm{I} .5$ minutes in phase angle. In nearly all practical cases the inaccuracy of the temperature measurements alone is sufficient to cause an error as great as the combined approximations, to say nothing of instrumental errors and the assumption that the reactance under working conditions is the same as that under short circuit conditions.

It is evident from the form of the equations that when plotted against current at a given power factor and voltage the phase angle of a transformer gives a straight line. The ratio and regulation give nearly a straight line, the very slight curvature being due to the square term. With constant secondary current, the phase angle gives a sine curve when plotted against the phase angle of the connected load, crossing the axis of zero phase near the point where $\theta=\tan ^{-1} \frac{X}{R}$. Similarly, the ratio and regulation give nearly sine curves (distorted by the square term) which have their maxima where $\theta=\tan ^{-1} \frac{X}{R}$. Theserelations may be found useful even when the short-circuit data are not available. 
A question has been raised in regard to the exact definition of rated current and rated voltage of a transformer when the voltage drop within the windings is taken into consideration. It appears that different engineers give different interpretations of the Standardization Rules of the American Institute of Electrical Engineers covering the point, viz: Rules 65, 65a, 67, 68, 69, 7o, and 197. Three interpretations may be given, as may be most clearly seen by a particular case, say a $5 \mathrm{kva}, 2200 / \mathrm{I}$ Io volt transformer.

First. Hold 2200 volts on the primary. Draw 5000/1 Io amperes. Take the reading of a voltmeter placed on the secondary as $\mathrm{E}_{2}$.

Second. Hold 2200 volts on the primary. . Draw 5000 volt amperes from the secondary. Take the readings of voltmeter and ammeter placed in the secondary as $\mathrm{E}_{2}$ and $\mathrm{I}_{2}$.

Third. Apply whatever primary voltage is necessary to make $E_{2}=110$ when 5000 volt-amperes are being drawn.

Since the ratio given on the name plate of power transformers is the ratio of turns and not of full-load terminal voltages, these interpretations give slightly different values. While the magnitude involved is small, it is a matter on which the Institute Rules should be definite, since it affects all the factors involved in the rating of transformers. It is to be hoped that the ambiguity will be removed in the next revision.

The third interpretation would seem to be preferable, since rule 65 , while applying primarily to power, seems, at least by implication, to indicate that rated voltage is the secondary terminal voltage at rated secondary kva load. We find that this is the interpretation of a number of engineers.

\section{EXPERIMENTAL TESTS OF THE FORMULAS}

As a test of the formulas accurate measurements were made of ratio and phase angle of two different types of lighting transformers by the potentiometer method ${ }^{4}$ and covering the whole range of power factors from zero power-factor current leading to zero power-factor current lagging.

\footnotetext{
1 For a description of this method, see Agnew and Silsbee, Proceedings A. I. E. E., 31, 1267; June, 1912.
} 
These phase relations were obtained by opposing two similar transformers as in the usual connections for a heat run. The emf which was inserted to circulate the load current was derived from the secondary of a phase shifting transformer excited from a second generator on the same shaft as that which excited the transformer under test. The magnitude of the current could be controlled by the generator field and its phase adjusted independently by the position of the rotor of the phase-shifting transformer. The temperature was maintained very nearly constant during the measurements, and the results have been corrected for the slight variations which did occur. As the transformers were small, it was necessary to correct for losses in the measuring instruments employed.

The impedances were determined by the usual short-circuit test using carefully calibrated portable instruments. In both transformers the resistance was found to be the same on a. c. as on $d$. c. and the leakage reactance appeared independent of the current up to double-rated current.

Data on Transformers

\begin{tabular}{|c|c|c|c|c|c|}
\hline & $\begin{array}{c}\text { Trans- } \\
\text { former A }\end{array}$ & $\begin{array}{l}\text { Trans- } \\
\text { former B }\end{array}$ & & $\begin{array}{l}\text { Trans- } \\
\text { former A }\end{array}$ & $\begin{array}{l}\text { Trans- } \\
\text { former B }\end{array}$ \\
\hline Rated kva ............ & 0.6 & 5.0 & \multirow{8}{*}{ 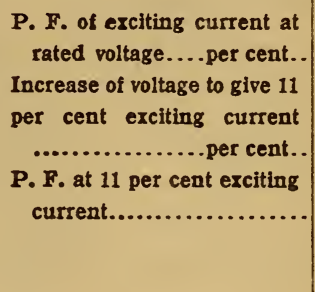 } & \multirow{8}{*}{0.39} & \multirow{4}{*}{0.23} \\
\hline Frequency.............. & 60.0 & 60.0 & & & \\
\hline Voltage.............. & $240 / 120$ & $1100 / 110$ & & & \\
\hline Method of cooling. ........... & Air only & Oil & & & \\
\hline Resistance drop....per cent.. & 2.3 & 2.0 & & & \multirow[t]{2}{*}{23.0} \\
\hline Reactance drop........ do.... & 1.6 & 1.0 & & & \\
\hline Impedance drop.......do.... & 2.8 & 2.2 & & & 0.09 \\
\hline $\begin{array}{l}\text { Exciting current at rated } \\
\text { voltage.......... per cent. }\end{array}$ & 11.0 & 3.4 & & & \\
\hline
\end{tabular}

Figs. 2 to 5 have been plotted to show the results of the comparison. Ratio, regulation, and phase angle are plotted against the angle between secondary current and voltage. (Power factors have also been indicated on the axis of abscissas.) The full lines are computed by the formulas from the short-circuit data, while the circles represent observed points. It will be seen that the agreement is quite satisfactory. In the runs plotted 


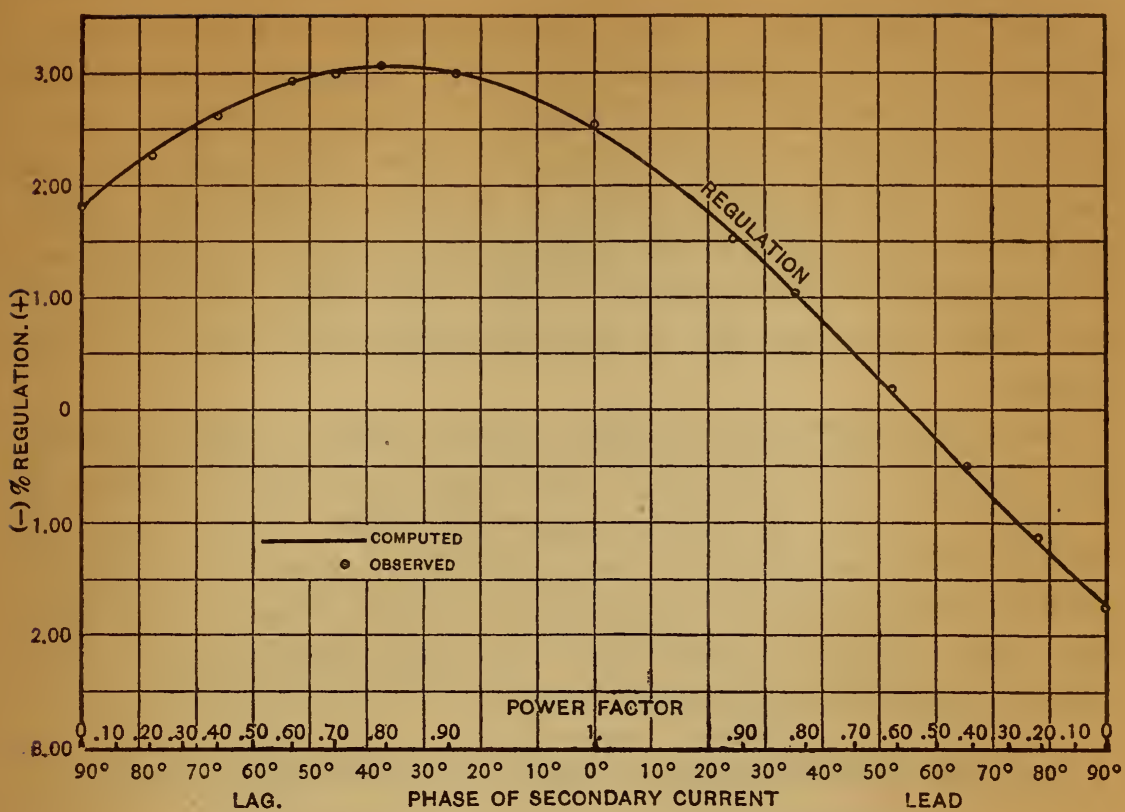

Fig. 2.-Regulation of a $0.6 \mathrm{kva}$. $240 / \mathrm{I} 20$ volt transformer

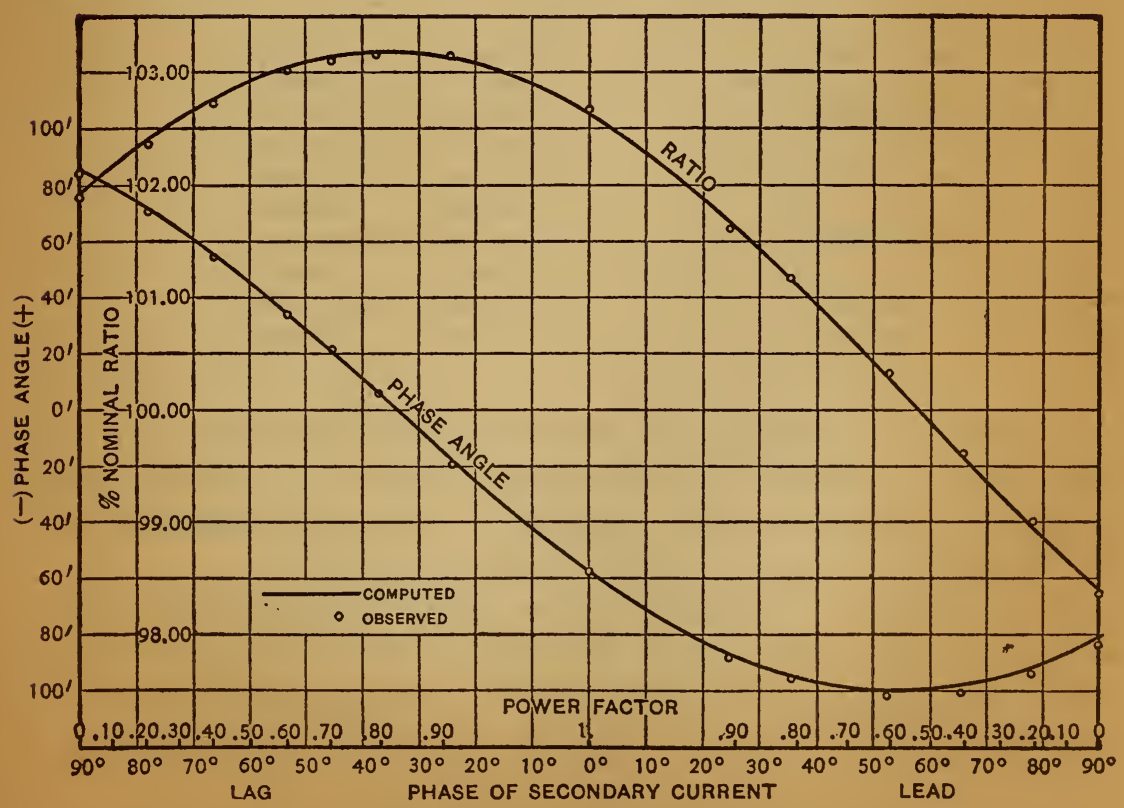

Fig. 3.-Ratio and phase angle of a 0.6 kva. $240 / 120$ volt transformer 
in Figs. 6 and 7 the voltage on transformer B was arbitrarily raised to give an exciting current of II per cent of rated load current, so as to magnify the effect of the approximations made in the derivation of the formulas. The agreement between computed and observed values is entirely satisfactory, again showing that the effect of the exciting current upon the regulation is inappreciable. The current rating was taken as unchanged and hence the regulation is proportionately less than at rated voltage.

The average differences (taken without regard to sign) between the computed and observed values for the various groups of readings are given in the accompanying table. (Since they are averages they are carried to one place farther than the sensitivity of the method of direct measurement.) The average differences taken with regard to sign were negligible, showing that there were no appreciable systematic errors.

Average Difference between Observed and Computed Values.

\begin{tabular}{|c|c|c|c|c|c|}
\hline & $\begin{array}{l}\text { Trans- } \\
\text { former A }\end{array}$ & $\begin{array}{c}\text { Trans- } \\
\text { former B }\end{array}$ & & $\begin{array}{c}\text { Trans- } \\
\text { former A }\end{array}$ & $\begin{array}{c}\text { Trans- } \\
\text { former B }\end{array}$ \\
\hline 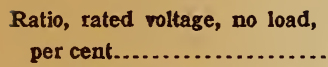 & 0.006 & 0.010 & $\begin{array}{l}\text { Regulation, excess voltage, full } \\
\text { load................ per cent. }\end{array}$ & & 0.010 \\
\hline 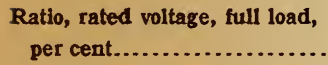 & .021 & .022 & $\begin{array}{c}\text { Phase angle, rated voltage, no } \\
\text { load.............. minutes.. }\end{array}$ & 0.9 & .1 \\
\hline $\begin{array}{l}\text { Ratio, excess voltage, no load, } \\
\text { per cent.......................... }\end{array}$ & & .005 & $\begin{array}{l}\text { Phase angle, rated voltage, } \\
\text { full load............ minutes.. }\end{array}$ & 1.3 & .8 \\
\hline $\begin{array}{l}\text { Ratio, excess voltage, full load, } \\
\text { per cent...................... }\end{array}$ & & .010 & $\begin{array}{l}\text { Phase angle, excess voltage, } \\
\text { no load........... minutes.. }\end{array}$ & & .1 \\
\hline $\begin{array}{l}\text { Regulation, rated voltage, full } \\
\text { load............... per cent.. }\end{array}$ & .016 & .015 & $\begin{array}{l}\text { Phase angle, excess voltage, } \\
\text { full load........... minutes.. }\end{array}$ & & 1.6 \\
\hline
\end{tabular}

As would be expected, the agreement at no load is better than with load. It should be remembered that the no-load phase angle is computed on the assumption that the reactance drop of the primary is equal to that of the secondary.

It is evident, at least for the two types of transformers represented in these tests, that the short-circuit test gives results which are entirely adequate for the predetermination of the

5 For further experimental evidence on this point, see L,loyd and Agnew, this Bulletin, 6, p. 273. Reprint No. 129. 


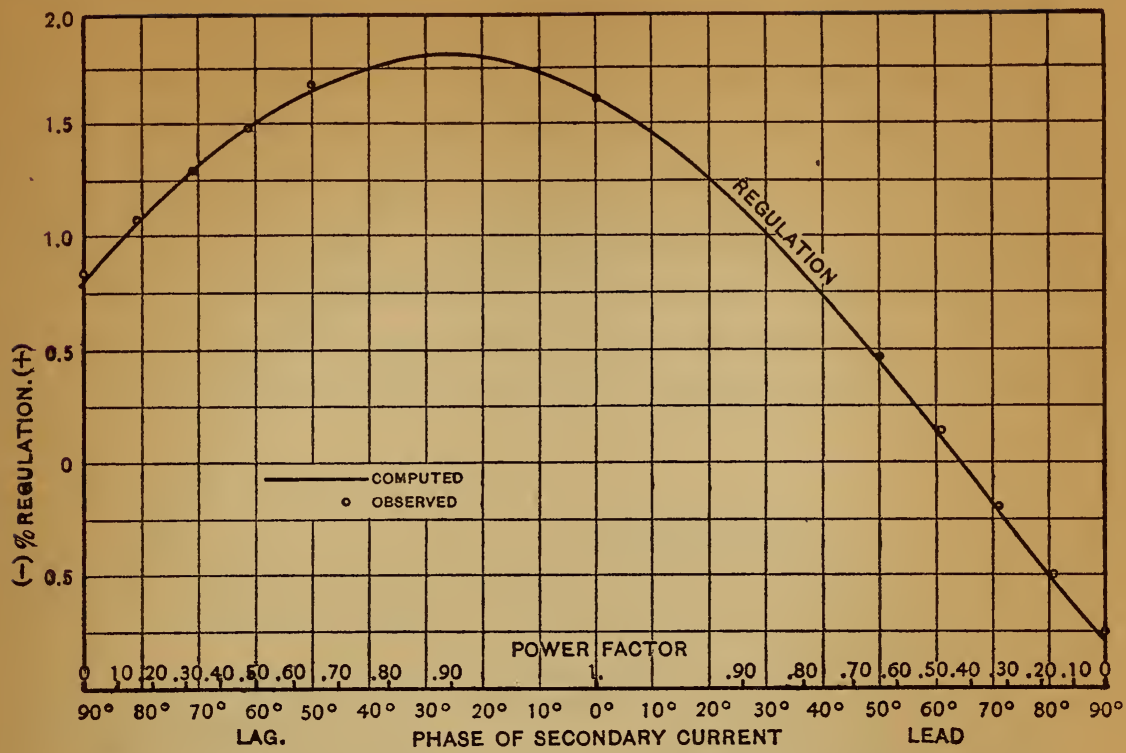

Fig. 4. - Regulation of a 5 kva. IIOO/IIO volt transformer at normal voltage

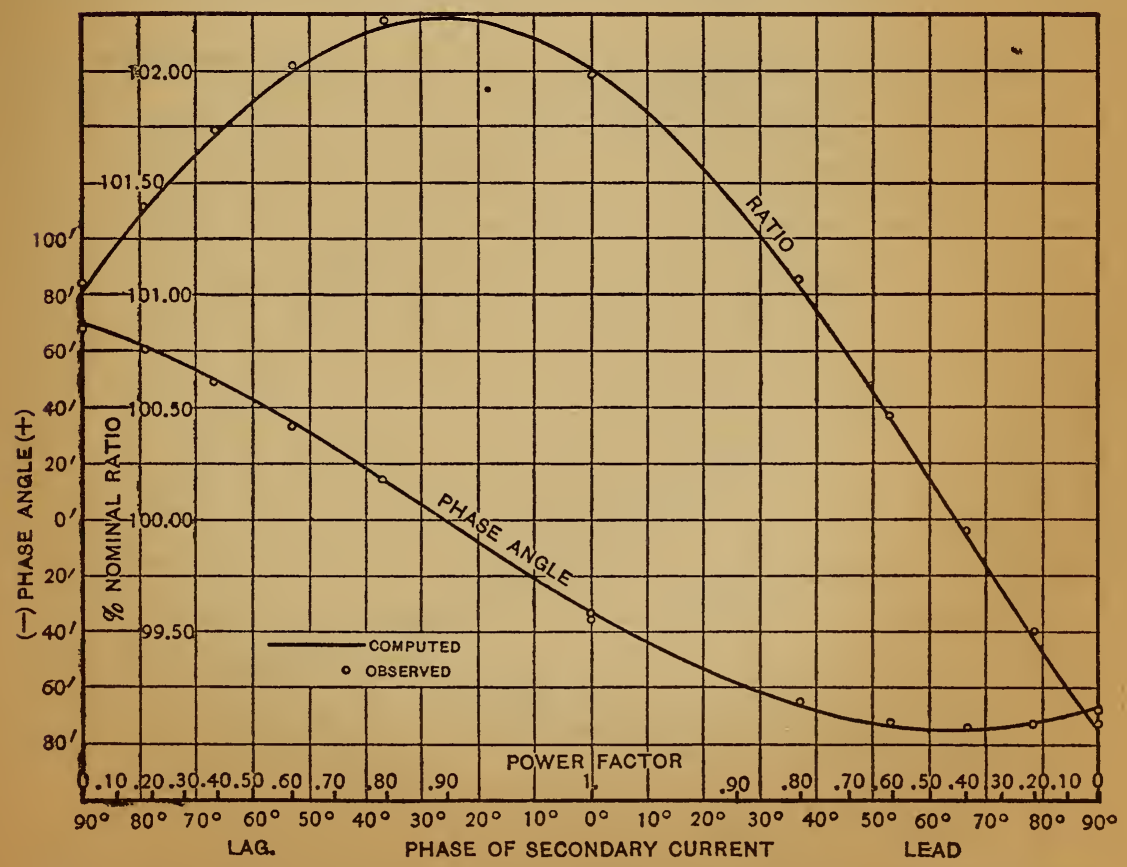

Fig. 5.-Ratio and phase angle of a 5 kva. IIOO/IIO volt transformer at normal voltage 


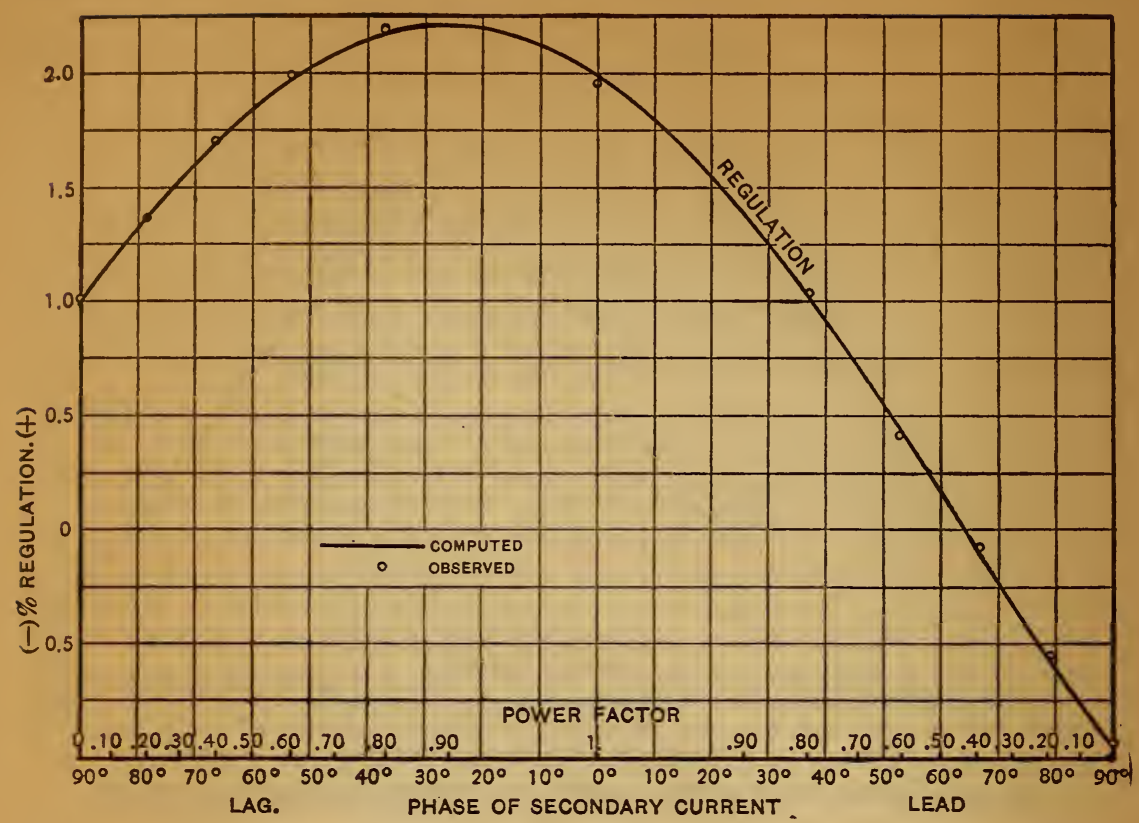

Fig. 6. - Ratio and phase angle of a $5 \mathrm{kva}$. IIOO/IIO volt transformer at excess voltage

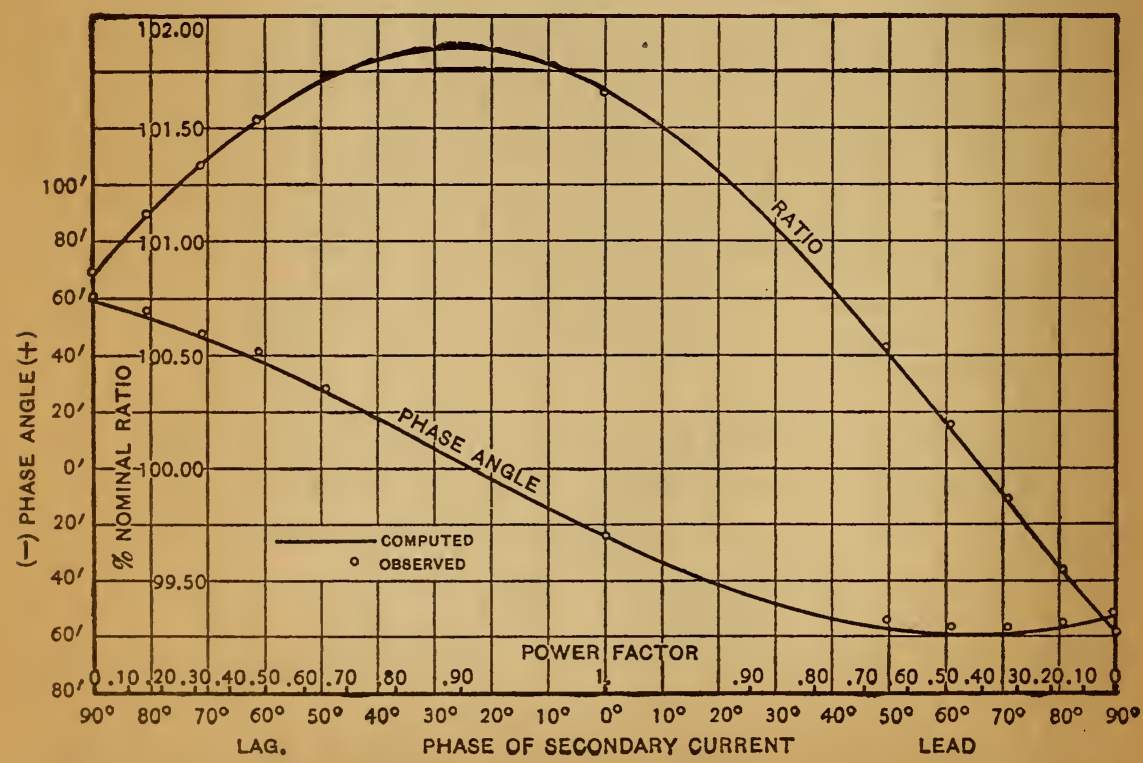

Fig. 7.-Ratio and phase angle of a $5 \mathrm{kva}$. $1100 / \mathrm{I} 10$ volt transformer at excess voltage 
operating characteristics, the accuracy being limited by the errors of measurement and not by theoretical errors.

The applications of these formulas to engineering problems do not come within the scope of this paper. ${ }^{6}$

It is to be hoped that tests along the lines of those here described may be carried out with widely differing types and sizes of transformers by laboratories, such as those of the manufacturing companies, which have the necessary facilities, in order that the adequacy or the limitations of the formulas may be definitely settled.

WASHINGTON, July II, 19I3.

NOTE.-Since the foregoing was written the attention of the standardization committee of the American Institute of Electrical Engineers has been called to the ambiguity in the interpretation of Rules $65,65 \mathrm{a}$, $67,68,69,70$, and 197 , and they have officially decided upon the third interpretation given.

- It may be well to mention a point which is sometimes overlooked in paralleling transformers. Two transformers may have the same regulation and the same no-load ratio and still, when connected in parallel, have a very considerable circulating current caused by inequality of phase angle. 


